\title{
La transplantation hépatique \\ chez les patients \\ co-infectés \\ VIH/VHC
}

> L'infection par le virus de l'immunodéficience humaine (VIH) a longtemps été considérée comme une contre-indication à la transplantation hépatique. Les raisons en étaient le pronostic sombre lié à la maladie VIH. L'avènement des trithérapies antirétrovirales a révolutionné le traitement des patients infectés par le VIH. Trente pour cent et $10 \%$ des patients infectés par le VIH sont également infectés respectivement par le virus de l'hépatite C (VHC) et par le virus de l'hépatite B (VHB). L’hépatite chronique $C$ et $B$ semble progresser plus vite chez les patients co-infectés et un nombre important de patients développent une cirrhose menaçant le pronostic vital. La transplantation hépatique pose plusieurs problèmes dans ce contexte: (1) le risque d'accident d'exposition au sang lors de cette intervention longue et hémorragique; (2) le moment de l'indication de la transplantation; (3) I'interférence entre les antirétroviraux et les inhibiteurs de la calcineurine; (4) le risque de récidive du VHB ou du VHC. Depuis 1999, un programme de transplantation hépatique chez les patients co-infectés a démarré avec le soutien de l'Agence nationale de recherche contre le sida et les hépatites. Les premiers résultats montrent une survie à 2 ans de $70 \%$ des patients infectés par le VHC et de $100 \%$ des patients infectés par le VHB. II n'a pas été noté de progression accélérée de la maladie VIH. La récidive virale B est bien prévenue par l'association post-transplantation d'immunoglobulines spécifiques anti-HBs et d'analogues nucléosidiques et nucléotidiques efficaces contre le VHB. La difficulté majeure est la récidive virale $C$. L'identification de ses mécanismes, sa prévention et son traitement sont les futurs défis à résoudre. <

Jean-Charles Duclos-Vallée, Elina Teicher, Daniel Vittecoq, Didier Samuel

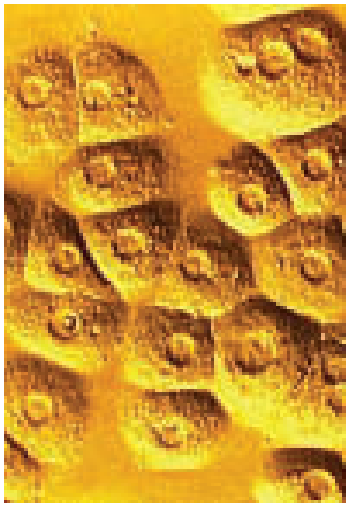

Les infections par le virus de l'hépatite $C$ (VHC) et le virus de l'immunodéficience humaine (VIH) sont des problèmes de santé publique majeurs. Plus de 40 millions de personnes sont infectés par le VIH dans le monde, et $2 \%$ à $3 \%$ de la population sont infectés par le virus de l'hépatite $C$ soit environ 175 millions de personnes. Dix millions d'individus sont co-infectés par le VIH et le VHC. La transmission de l'infection par le VHC, comme celle du VIH, s'effectue majoritairement par voie parentérale. Ainsi la co-infection VIH/VHC est volontiers fréquente dans la population toxicomane et celle des hémophiles. Une infection par le virus de l'hépatite C est présente chez $70 \%$ à $90 \%$ de la population hémophile et $60 \%$ à $80 \%$ de la population toxicomane.

Si l'introduction des thérapies antirétrovirales a transformé le pronostic des patients infectés par le VIH, il est admis que l'infection par le VHC représente la première cause de morbidité et de mortalité dans cette population.

La cinétique de progression de la fibrose hépatique est particulièrement rapide chez les patients co-infectés VIH/ VHC, et 15 à $25 \%$ de ces patients développent une cirrhose après 10 à 15 ans d'évolution [1-5] (Figure 1). Cette évolu- 


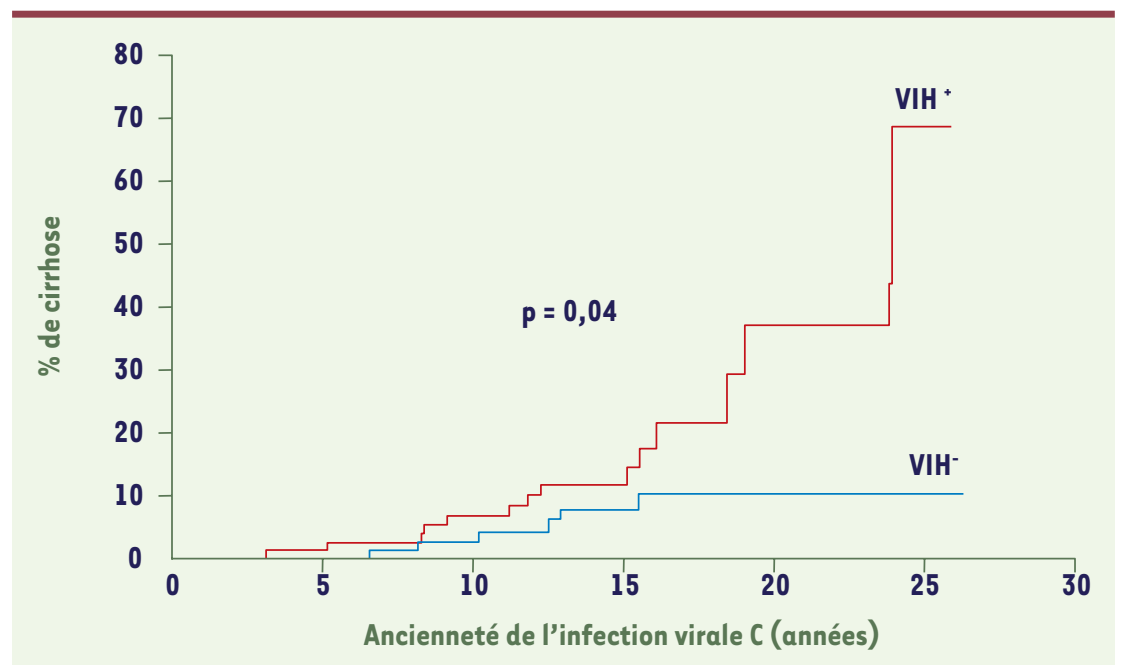

Figure 1. Pourcentage de patients co-infectés VIH/VHC atteints de cirrhose selon l'ancienneté de l'infection virale C. La progression vers la cirrhose est significativement plus rapide chez les patients co-infectés (d'après [3]).

tion sévère est aussi très liée à l'état d'immunodépression et en particulier à la baisse du taux de lymphocytes T CD4 (<200 cellules $\left./ \mathrm{mm}^{3}\right)$ [6]. Ainsi les taux de cirrhose décompensée et de mortalité lié à la maladie hépatique dans la population co-infectée VIH/VHC sont élevés : la proportion de décès attribuable à la cirrhose et/ou au développement d'un carcinome hépatocellulaire s'est accru de $1,5 \%$ à $14,3 \%$ [7, 8]. Selon Pineda et al., la médiane de survie après un premier épisode de décompensation de la cirrhose est de 16 mois [9] (Figure 2). De même, le taux de mortalité est de 14,2/1 000 chez les patients co-infectés VIH/VHB, tandis qu'il n'est que de 0,8/1 000 chez les patients mono-infectés par le VHB [10]. Le carcinome hépatocellulaire ou cancer primitif du foie, est diagnostiqué à un âge plus jeune, et à un stade plus avancé chez les patients co-infectés VIH/VHB que dans la population mono-infectée, avec pour conséquence une survie plus courte [11]. En France, l'incidence annuelle du carcinome hépatocellulaire est 5 fois plus élevée dans la population co-infectée VIH/VHC que dans la population mono-infectée VHC [8].
On comprend ainsi que l'indication de la transplantation hépatique puisse concerner les patients infectés par le VIH et en particulier ceux qui sont co-infectés par le VHC. Cette mise au point a pour but de faire le point sur les indications et les résultats de la transplantation hépatique chez les patients co-infectés VIH/VHC et VIH/VHB.

\section{Quelles sont les indications de la transplantation hépatique?}

Les indications de la transplantation hépatique chez les patients co-infectés reposent sur la présence d'une hépatopathie sévère qui menace le pronostic vital dans les 2-3 ans et/ ou d'un nodule unique de carcinome hépatocellulaire de taille inférieure à $5 \mathrm{~cm}$ ou la présence de moins de 3 nodules d'une taille inférieure à $3 \mathrm{~cm}$. Des scores de gravité tels que le MELD (model for end stage liver disease) ${ }^{1}$ score qui tient compte de I'INR (international normalized ratio), de la bilirubine et de la créatininémie, et le score de Child-Pugh (trois catégories A, B et C) qui tient du compte de la présence d'une ascite, du degré d'encéphalopathie, du taux de bilirubine et du taux de prothrombine, ne semblent pas adaptés pour décider de l'indication d'une transplantation hépatique dans la population co-infectée $[12,13]$. $\varepsilon n$ effet, chez les patients VIH/VHC, l'évolution est volontiers rapide et de ce fait, adresser un patient à un centre de transplantation selon les critères classiques de sévérité peut se révéler une décision trop tardive. Il est impératif de définir, chez les patients co-infectés, des paramètres pronostiques spécifiques à ce type de pathologie et de pouvoir les reproduire dans le but de définir au mieux le moment pour démarrer un bilan de transplantation en prenant en compte, bien sûr, le délai d'attente [13]. Dans l'état actuel des choses, les patients avec un score de Child-Pugh B et a fortiori C doivent être adressés à un centre de greffe. Dans notre expérience, des éléments comme la présence d'une ascite et/ou d'une encéphalopathie

${ }^{1}$ Score de MELD : $9.57 \times \log _{\mathrm{e}}($ créatinine $\mathrm{mg} / \mathrm{dl})+3.78 \times \log _{\mathrm{e}}$ bilirubine totale $\mathrm{mg} / \mathrm{dl})+11.20 \times \log _{\mathrm{e}}(\mathrm{INR})+6.43$ (constant quelle que soit l'étiologie de la maladie hépatique). 
lors de la première consultation dans notre centre sont des facteurs indépendants de survie pris en considération pour l'inscription sur la liste d'attente. En ce qui concerne les patients co-infectés VIH/VHB, I'utilisation de combinaisons de molécules antivirales (Lamivudine-Tenofovir, FTC-Tenofovir), rapidement efficaces sur la réplication du VHB et du VIH permettent dans la très grande majorité des cas de contrôler la maladie hépatique. Ainsi, la co-

infection VIH/VHB constitue à l'heure actuelle une indication plus rare de transplantation hépatique réservée essentiellement aux patients atteints de carcinome hépatocellulaire.

\section{Les aspects particuliers \\ de la transplantation hépatique chez les patients co-infectés}

Dans la période suivant la greffe, deux problèmes majeurs surviennent représentés d'une part par la toxicité des antirétroviraux, et d'autre part la récidive virale $C$ sur le greffon (Tableau I) [14-22].

\section{Cause de mortalité/délai post-transplantation} Nombre de patients

VHC : 8

VHB/VHC : 2

États-Unis $\mathrm{VHB}: 2$ $n=2$

Sepsis : $\mathbf{1 2}$

[15] Hépatite fulminante VHB : 2 Hépatite fulminante : 2

Récidive virale C : J570
VHC : 7

Grande-

Bretagne

[19]

VHB : 4

Hépatite fulminante: 1

Cirrhose alcoolique: 2

\section{$n=5$}

Rupture anévrisme cérébral

et récidive virale $C: n=1$

Récidive virale $C: n=4$

VHC : 4

Allemagne

[21]

VHB : 1

Hépatite fulminante HBV : 2

$n=1$

Hémorragie intrathoracique : 195

Espagne $\quad \mathrm{VHC}: 3$

[22]

VHC + VHB + virus Delta : 1

$\mathrm{n}=1$

Récidive virale C : M17

\begin{tabular}{|c|c|c|c|}
\hline $\begin{array}{c}\text { France } \\
{[18]}\end{array}$ & VHC : 7 & $n=2$ & $\begin{array}{l}\text { Récidive VHC } \\
\text { et toxicité mitochondriale : M4 et M24 }\end{array}$ \\
\hline $\begin{array}{c}\text { France } \\
{[24]}\end{array}$ & VHC : 23 & $n=6$ & $\begin{array}{l}\text { Hémorragie cérébrale : } M 3 \\
\text { Récidive VHC et toxicité mitochondriale: } \\
\mathrm{n}=2(M 4, M 24) \\
\text { Infection pulmonaire : M6 } \\
\text { Cancer du pancréas : } M 18 \\
\text { Récidive virale } C: M 27\end{array}$ \\
\hline $\begin{array}{l}\text { États-Unis } \\
\text { [25] }\end{array}$ & VHC : 27 & $n=13$ & $\begin{array}{l}\text { Récidive virale } C: n=6 \\
(M 49, M 14, M 9, M 11, M 13, M 4) \\
\text { Récidive virale } C \text { et rejet chronique }: n=1(M 20) \\
\text { Défaillance multiviscérale }: n=2(S 1, S 2) \\
\text { Récidive carcinome hépatocellulaire }: n=1(M 12) \\
\text { Sepsis : } n=3(M 2, M 3, M 6)\end{array}$ \\
\hline
\end{tabular}

Tableau I. Principales expériences de transplantation hépatique chez les patients co-infectés par le VIH dans la littérature.

\section{La toxicité}

des antirétroviraux

sur le greffon

Il est bien admis que les trois classes d'antirétroviraux (analogues nucléosidiques, inhibiteurs de protéase et analogues non nucléosidiques) sont susceptibles d'induire une toxicité hépatique. Les analogues nucléosidiques comme la zidovudine, la stavudine et la didanosine peuvent provoquer la survenue d'une stéatose microvésiculaire avec acidose lactique. De même, la toxicité des antirétroviraux sur le greffon peut être potentiellement sévère; ainsi, parmi les sept premiers malades transplantés dans notre centre, nous avons observé deux cas de toxicité mitochondriale sévère sur le greffon responsable de leur décès. Ces deux patients étaient traités par analogues nucléosidiques. Cette hépatotoxicité est liée à une toxicité mitochondriale, elle-même due à l'inhibition de la $\gamma$ ADN polymérase [23]. En culture cellulaire, la toxicité mitochondriale des 
inhibiteurs nucléosidiques de la transcriptase inverse est ainsi hiérarchisée : zalcitabine > stavudine > zidovudine > didanosine > lamivudine > abacavir [23].

Il est très vraisemblable que l'infection virale $C$, ou plutôt la récidive de l'infection virale $C$, potentialise la toxicité mitochondriale des antirétroviraux. En effet, d'une part, une stéatose peut être associée à l'infection virale C indépendamment de l'infection VIH, d'autre part ce type de toxicité des antirétroviraux n'est pas observé après greffe chez les patients co-infectés VIH/VHB.

Un autre type de toxicité ne doit pas être négligé en période postgreffe: il s'agit de la toxicité pancréatique des antirétroviraux. Nous avons constaté la survenue de 3 cas de pancréatite aiguë dont un cas de pancréatite aiguë sévère dans notre expérience de 23 patients transplantés. Ce type de toxicité était vraisemblablement imputable à l'administration d'analogues nucléosidiques.

\section{La récidive virale $\mathrm{C}$ sur le greffon}

La récidive virale $C$ sur le greffon est constante et volontiers sévère et se manifeste par l'apparition d'une hépatite chronique caractérisée par une fibrose rapidement évolutive. Nous avons en effet montré que les patients transplantés co-infectés VIH/VHC présentaient plus fréquemment, 6 mois après la greffe, une fibrose sévère voire une cirrhose. Parmi les vingt-trois premiers patients co-infectés transplantés dans notre centre, cinq (22\%) ont développé une fibrose sévère, classée F3 dans le score METAVIR et deux (9\%) ont développé une cirrhose [24]. La vitesse de fibrose calculée à partir du taux de fibrose, rapportée au temps écoulé depuis la greffe est significativement plus élevée dans la population coinfectée que dans la population mono-infectée. En ce qui concerne la charge virale $C$, celle-ci, calculée à 6 mois post-transplantation est significativement plus élevée dans la population co-infectée

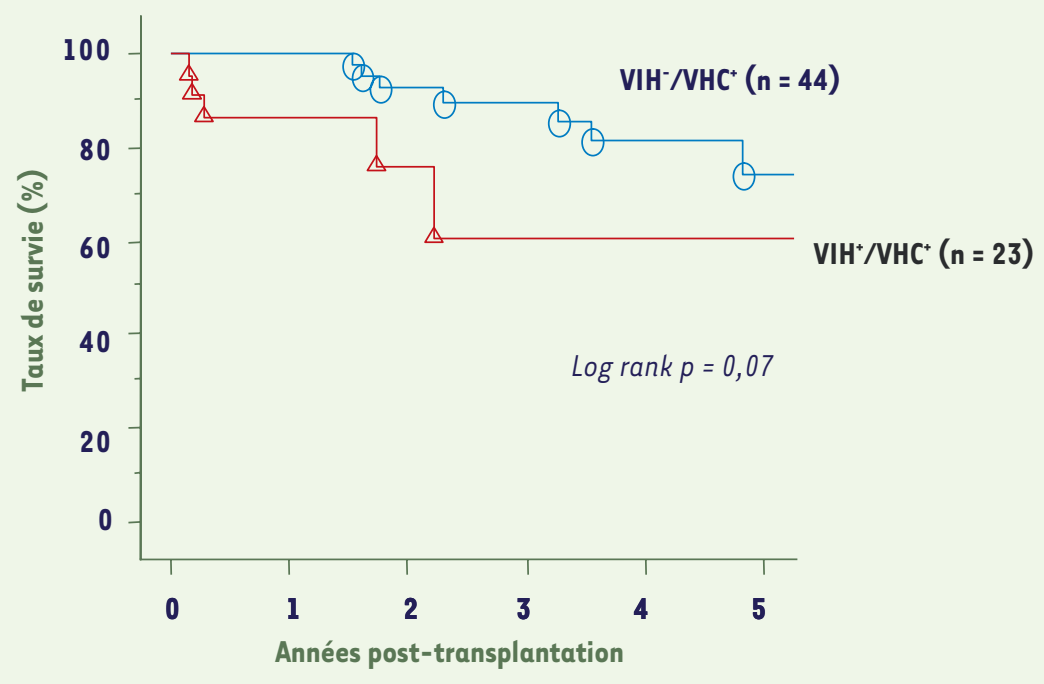

Figure 3. Survie actuarielle de 23 patients co-infectés $\mathrm{VIH}^{+} / \mathrm{VHC}^{+}$et 44 patients $\mathrm{VIH}^{-} / \mathrm{VHC}^{+}$après transplantation hépatique à l'hôpital Paul Brousse. Le taux de survie à 2 ans après transplantation dans le groupe $\mathrm{VIH}^{+} / \mathrm{VHC}^{+}$est de $72 \%$ et de $92 \%$ dans le groupe $\mathrm{VIH}^{-} / \mathrm{VHC}^{+}$. que dans la population mono-infectée. Ces constatations amènent à discuter l'indication d'un traitement antiviral $C$, administré précocement après la greffe, avec le double objectif d'obtenir une réponse virologique prolongée et d'éviter une progression de la fibrose sur le greffon.

\section{La transplantation}

chez les patients co-infectés VIH/VHB

La transplantation chez les patients co-infectés VIH/VHB est facilitée par le contrôle possible de la réplication virale $B$ avant la greffe et en période post-greffe. La réinfection virale $B$ du greffon est évitée par l'administration combinée, après la transplantation, de fortes doses d'immunoglobulines anti-HBs et d'analogues nucléos(t)idiques dirigés contre le VHB.

\section{Le problème de l'interaction entre les traitements} antirétroviraux et les inhibiteurs de la calcineurine La ciclosporine, le tacrolimus et la rapamycine sont métabolisés par les cytochromes P4503A2 et 3A4 et sont donc susceptibles d'être la cible d'interactions pharmacocinétiques avec d'autres drogues qui induisent ou inhibent ces activités enzymatiques. Ces agents sont aussi le substrat du système glycoprotéine $P$ (une pompe qui limite l'absorption intracellulaire et favorise l'élimination de substances exogènes). Les inhibiteurs de protéase sont métabolisés par ces mêmes systèmes enzymatiques et agissent soit comme inducteurs, soit comme inhibiteurs de ces voies métaboliques. Au moment de la réintroduction des inhibiteurs de protéases, un surdosage en immunosuppresseurs responsable d'une toxicité essentiellement rénale peut donc survenir du fait de l'inhibition du métabolisme des anticalcineurines; il faut donc être très vigilant et, au moment de cette réintroduction, baisser les doses d'immunosuppresseurs qui doivent être ajustées selon un monitorage quotidien.

\section{Les complications infectieuses}

Nous n'avons pas observé, après transplantation, plus de complications infectieuses chez les patients co-infectés que chez les patients mono-infectés par le virus de l'hépatite C. En ce qui concerne les infections opportunistes, une pneumopathie à cytomégalovirus 
ainsi qu'une œsophagite à Candida ont été observées chez les patients co-infectés VIH/VHC. II n'y a pas d'éléments dans notre série ou dans celles de la littérature pour suggérer une accélération de la maladie VIH après la transplantation hépatique $[15,16]$. Le chiffre absolu de CD4 reste stable ou augmente après la transplantation.

\section{La survie post-greffe}

Dans l'expérience nord-américaine rapportée récemment, la survie, bien qu'inférieure, n'était pas significativement différente chez les patients co-infectés transplantés et chez les patients infectés VHC sans infection VIH transplantés pour cirrhose virale C; la survie à 1 an, 3 ans et 5 ans était de $67 \%, 56 \%$ et de $33 \%(p=0,07)$ dans le groupe co-infecté et de $76 \%$, $72 \%$ et de $72 \%$ dans le groupe mono-infecté [25]. Dans une étude menée par le même groupe, la survie était influencée par la toxicité des antirétroviraux, un taux de CD4 après greffe inférieur à 200 cellules/ $\mu$ et une charge virale VIH post-transplantation supérieure à 400 copies/ml [26].

Dans notre expérience, la survie des patients co-infectés VIH/VHC à 2 ans est de $70 \%$ et le seul facteur pronostic influençant la survie est le score MELD calculé avant greffe (Figure 3).

La survie après greffe des patients co-infectés VIH/ VHB est de $100 \%$ à 2 ans ce qui confirme que ce type de transplantation donne des résultats équivalents à la transplantation chez les patients mono-infectés VHB.

\section{Conclusions}

La transplantation hépatique des malades infectés par le VIH pose un problème de fond car il s'agit d'une indication nouvelle dans un contexte de pénurie d'organes. Nous avons lancé l'expérience dans notre centre depuis plus de 5 ans avec près de 50 patients transplantés depuis cette date. II s'agit d'une expérience difficile qui ne peut être menée que grâce à une collaboration très étroite entre hépatologues, chirurgiens, infectiologues, internistes, virologues, immunologistes et anatomo-pathologistes. II s'agit d'un progrès médical considérable; si des problèmes importants subsistent, comme la sévérité de l'infection virale $C$ sur le greffon, les résultats positifs obtenus nous incitent à développer ce programme. Une meilleure gestion des antirétroviraux, et l'avènement d'une nouvelle génération de traitements antiviraux contre l'hépatite $C$ permettront d'améliorer significativement les résultats. $\diamond$

\section{SUMMARY}

Liver transplantation for patients infected with both HIV and HCV or HIV and HBV

Human immunodeficiency virus infection (HIV) has been considered until recently as a contraindication for liver transplantation. This was due to the poor spontaneous prognosis of HIV infection. The advent of highly active antiretroviral drugs (HAART) was a therapeutic breakthrough, and the prognosis has been dramatically improved. $30 \%$ and $10 \%$ of HIV infected patients are coinfected with hepatitis $C$ virus (HCV) and with hepatitis B virus (HBV), respectively. The progression of chronic hepatitis $B$ and $C$ seems more rapid in coinfected patients, and a high number of patients will develop life-threatening liver cirrhosis. There are numerous potential problems raised by liver transplantation in HIV infected patients: (1) the potential risk of needlestick injury during this type of hemorrhagic surgery at high risk of bleeding; (2) the timing for liver transplantation; (3) the risk of interference between HAART and calcineurin inhibitors; (4) The risk of HBV and HCV recurrence post-transplant. Since 1999, a program of liver transplantation has been started in patients coinfected with HIV and HBV or HCV with the support of the Agence Nationale de Recherche contre le Sida et les Hépatites virales (ANRS). The first results showed that liver transplantation in HIV-HCV and HIV-HBV infected patients is feasible, achieving 2-year survival of $70 \%$ and $100 \%$, respectively. There was no acceleration of HIV disease after transplantation. HBV recurrence was well prevented by the combination of anti-HBs immunoglobulins plus nucleoside and nucleotide analogues effective against HBV. The main problem is HCV recurrence, which is more rapid and more severe in HIV coinfected patients than in HCV monoinfected patients. Understanding HCV recurrence mechanisms, and preventing and treating of HCV recurrence are major future challenges. $\diamond$

\section{REMERCIEMENTS}

Les auteurs remercient les docteurs Daniel Azoulay, René Adam, Henri Bismuth, Denis Castaing, Cyrille Feray, Bruno Roche, Philippe Ichai, Faouzi Saliba, Thérésa Antonini, Eric Vibert, Luc Antoine Veilhan, Paola Andreani, Anne Marie RoqueAfonso, Elisabeth Dussaix, Mylène Sebagh, Marie-Pierre Bralet, Catherine Guettier, Colette Danet, Elisabeth Pasdeloup, Catherine Tanguy, Gael Berthelot ainsi que l'ensemble des médecins qui ont adressé les patients vers notre centre pour leur précieuse collaboration.

\section{RÉFÉRENCES}

1. Benhamou Y, Bochet M, Di Martino V, et al. Liver fibrosis progression in human immunodeficiency virus and hepatitis $C$ virus coinfected patients. The Multivirc group. Hepatology 1999 ; 30 : 1054-8.

2. Soto B, Sanchez-Quijano A, Rodrigo L, et al. Human immunodeficiency virus infection modifies the natural history of chronic parentarally-acquired hepatitis $C$ with an unusually rapid progression to cirrhosis. J Hepatol $1997 ; 26$ : 1-5.

3. Di Martino V, Ruffat P, Boyer N, et al. The influence of human immunodeficiency virus coinfection on chronic hepatitis $C$ in injection drug users: a long-term retrospective cohort study. Hepatology $2001 ; 34: 1193-9$.

4. Pol S, Lamorthe B, Trinh Thi N, et al. Retrospective analysis of the impact of HIV infection and alcohol use on chronic hepatitis C in a large cohort of drug users. J Hepatol 1998 ; $28: 945-50$.

5. Vallet-Pichard A, Pol S. Natural history and predictors of severity of chronic hepatitis $C$ virus (HCV) and human immunodeficiency virus (HIV) co-infection. J Hepatol 2006 ; 44 : S28-44. 
6. Rockstroh JK, Spengler U, Sudhop U, et al. Immunosuppression may lead to progression of hepatitis C associated liver disease in hemophiliacs coinfected with HIV. Am J Gastroenterol $1996 ; 91: 2563-8$

7. Cacoub P, Geffray L, Rosenthal $\varepsilon$, et al. Mortality among human immunodeficiency virusinfected patients with cirrhosis or hepatocellular carcinoma due to hepatitis $C$ virus in French departments internal medicine/infectious diseases, in 1995 and 1997. Clin Infect Dis 2001 32 : 1207-14

8. Rosenthal $\varepsilon$, Poirée M, Pradier C, et al. Mortality due to hepatitis C-related liver disease in HIV-infected patients in France (Mortavic 2001 study). AIDS 2001 ; 17 : 1803-9.

9. Pineda JA, Romero-Gomez M, Dfaz-Garcia F, et al. HIV coinfection shortens the survival of patients with hepatitis $C$ virus-related decompensated cirrhosis. Hepatology 2005 ; $41: 779-89$.

10. Thio CL, Seaberg EC, Skolasky R Jr, et al. HIV-1, hepatitis B virus and risk of liver-related mortality in the multicenter cohort study (MACS). Lancet $2002 ; 360: 1921-6$.

11. Puoti M, Bruno R, Soriano V, et al. Hepatocellular carcinoma in HIV-infected patients: epidemiological features, clinical presentation and outcome. AIDS $2004 ; 18: 2285-93$.

12. Terrault N, Carter JT, Carlson L, et al. Outcome of patients with hepatitis B virus and human immunodeficiency virus infections referred for liver transplantation. Liver Transpl 2006 ; $12: 801-7$

13. Samuel D, Duclos-Vallée JC. The difficult timing for liver transplantation in cirrhotic patients coinfected with HIV. In search for a prognosis score. Liver Transpl 2006 ; 12 : 699-701.

14. Prachalias AA, Pozniak A, Taylor C, et al. Liver transplantation in adults coinfected with HIV. Transplantation $2001 ; 72: 1684-8$.

15. Neff G, Bonham A, Tzakis A, et al. Orthotopic liver transplantation in patients with human immunodeficiency virus and end-stage liver disease. Liver Transpl $2003 ; 9: 239-47$.

16. Ragni MV, Belle SH, Im K, et al. Survival of human immunodeficiency virus-infected liver transplanted recipients. J Infect Dis 2003; 188 : 1412-20.

17. Samuel D, Duclos-Vallée JC, Teicher $\varepsilon$, Vittecoq D. Liver transplantation in patients with HIV infection. J Hepatol $2003 ; 39: 3-6$.

18. Duclos-Vallée JC, Vittecoq D, Teicher $\varepsilon$, et al. Hepatitis $C$ virus viral recurrence and liver mitochondrial damage after liver transplantation in HIV-HCV co-infected patients. J Hepato $2005 ; 42: 341-9$
19. Norris S, Taylor C, Muiesan P, et al. Outcomes of liver transplantation in HIV-infected individuals: the impact of HCV and HBV infection. Liver Transpl $2004 ; 10: 1271-8$

20. Radeke K, Fruhauf NR, Miller M, et al. Outcome after othotopic live transplantation in five HIV-infected patients with virus hepatitisinduced cirrhosis. Liver Int 2005; 25 : 101-8.

21. Vogel M, Voigt $\varepsilon$, Schäfer $N$, et al. Orthotpic liver transplantation in human immunodeficiency virus (HIV)-positive patients: outcome of the 7 patients from the Bonn cohort. Liver Transpl 2005; 11 : 1515-21.

22. Moreno S, Fortun J, Quereda C, et al. Liver transplantation in HIVinfected recipients. Liver Transpl $2005 ; 11: 76-81$.

23. Nunez M. Hepatotoxicity of antiretrovirals: incidence, mechanisms and management. J Hepatol 2006 ; 44 : S132-9.

24. Duclos-Vallée JC, Feray C, Sebagh M, et al. Liver transplantation of HIVHCV and HIV-HBV coinfected patients: a large experience in a single centre. J Hepatol 2006 ; 44 : S2-8.

25. De Vera ME, Dvorchik I, Tom K, et al. Survival of liver transplant patients coinfected with HIV and HCV is adversely impacted by recurrent hepatitis C. Am J Transpl 2006; 6: 2983-93.

26. Ragni MV, Belle SH, Im K, et al. Survival of human immunodeficiency virus-infected liver transplant recipients. J Infect Dis 2003; 188: 1405-11
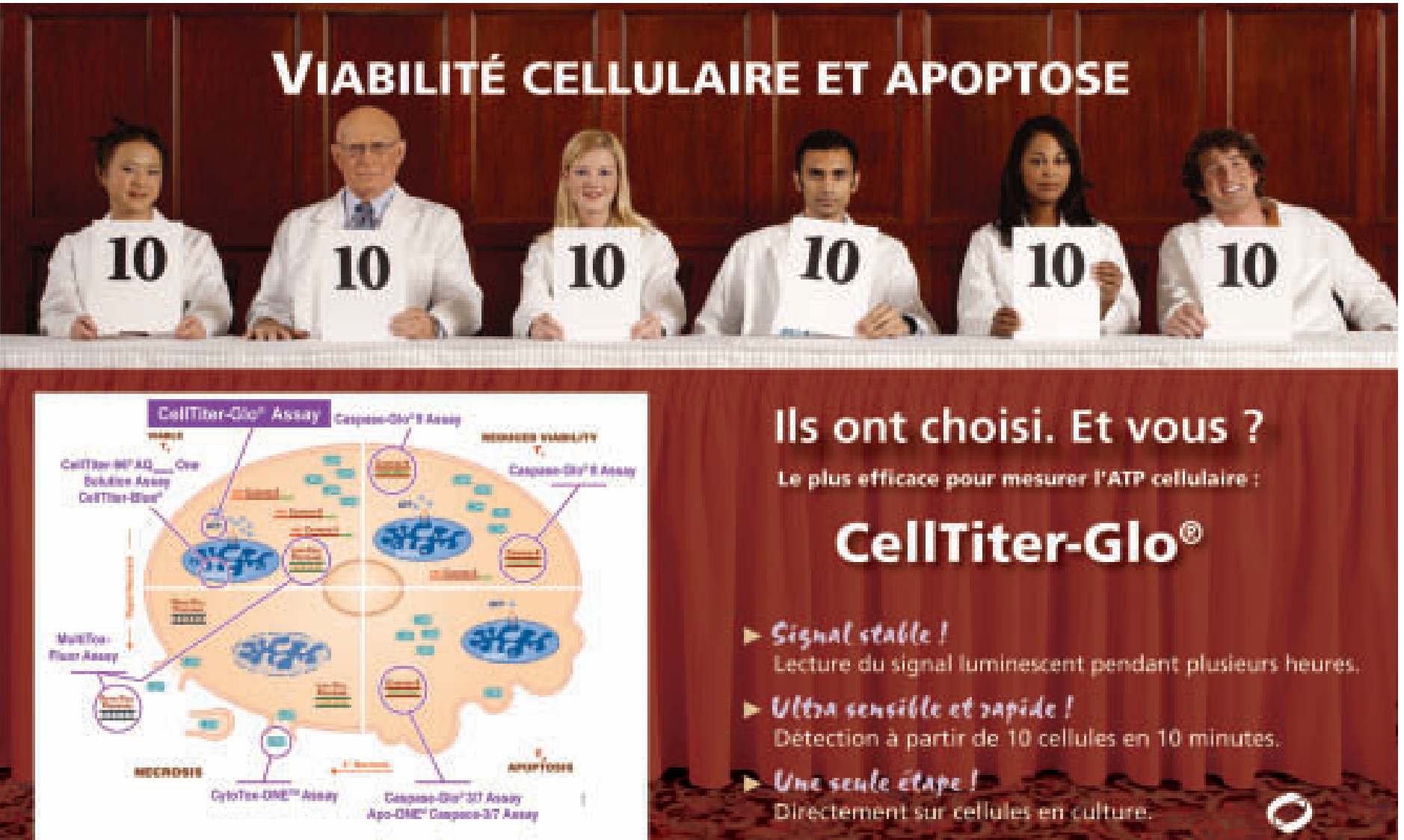

Ils ont choisi. Et vous ?

Le plus efficace pour mesurer I'ATP cellulaire :

\section{CellTiter-Glo ${ }^{\oplus}$}

